Check for updates

Cite this: Chem. Commun., 2020,

56, 13971

Received 12th June 2020

Accepted 13th October 2020

DOI: $10.1039 / \mathrm{d} 0 \mathrm{cc} 04116 \mathrm{e}$

rsc.li/chemcomm

\section{Unravelling a long-lived ligand-to-metal cluster charge transfer state in Ce-TCPP metal organic frameworks $\dagger$}

\author{
Sizhuo Yang, ${ }^{a}$ Wenhui Hu, ${ }^{a}$ James Nyakuchena, ${ }^{a}$ Christian Fiankor, ${ }^{b}$ Cunming Liu, ${ }^{c}$ \\ Eli Diego Kinigstein, ${ }^{c}$ Jian Zhang, ${ }^{b}$ Xiaoyi Zhang ${ }^{c}$ and Jier Huang (D)*a
}

\begin{abstract}
Metal organic frameworks (MOFs) have emerged as promising photocatalytic materials for solar energy conversion. However, a fundamental understanding of light harvesting and charge separation (CS) dynamics in MOFs remains underexplored, yet they are key factors that determine the efficiency of photocatalysis. Herein, we report the design and CS dynamics of the Ce-TCPP MOF using ultrafast spectroscopic methods.
\end{abstract}

The direct conversion of water or $\mathrm{CO}_{2}$ by sunlight into fuel is a promising approach to address global energy and environmental issues. ${ }^{1}$ However, it remains a great challenge to drive such a reaction in an efficient way and an appropriate catalyst is highly desired to promote the reaction at a reasonable rate. As an emerging class of porous materials, metal organic frameworks (MOFs) offer a new opportunity by taking advantage of both homogenous and heterogeneous catalysis for photocatalysis. ${ }^{2}$ MOFs are created by assembling metal-containing secondary building units (SBUs) with organic linkers. ${ }^{3}$ With flexible metal SBUs and organic linkers, MOFs are able to integrate lightharvesting materials and catalysts into a single matrix. ${ }^{4}$ In addition, the high crystallinity and porous nature of MOFs may facilitate charge transport and diffusion of reactants during the photocatalytic reaction. ${ }^{5}$ Due to these reasons, a large number of MOFs with built-in photosensitizers (PSs) and molecular catalysts have been used for water splitting and $\mathrm{CO}_{2}$ reduction. ${ }^{6}$ However, the majority of these works are centered on their catalytic performance and stability rather than fundamental mechanism. The commonly accepted catalytic pathway initiates with light absorption by the PS, which is followed by charge separation (CS) events either through oxidative

\footnotetext{
${ }^{a}$ Department of Chemistry, Marquette University, Milwaukee, Wisconsin, 53201, USA.E-mail: jier.huang@marquette.edu

${ }^{b}$ Department of Chemistry, University of Nebraska-Lincoln, Lincoln, Nebraska, 68588, USA

${ }^{c}$ X-ray Science Division, Argonne National Laboratory, Argonne, Illinois, 60349, USA

$\dagger$ Electronic supplementary information (ESI) available: Experimental details, Fig. S1-S8, Tables S1-S3. See DOI: 10.1039/d0cc04116e
}

quenching of the PS by electron transfer (ET) to the catalyst or reductive quenching through ET from the electron donor. As a result, it is essential to gain thorough knowledge of the fundamental aspects of the light harvesting and CS processes before we explore MOFs for photocatalytic applications. ${ }^{6 c, 7}$ Indeed, the presence of a long-lived CS state in a few MOFs following photoexcitation has been reported previously. ${ }^{7 a, 8}$ We have also reported the formation of a long-lived CS state in a zeolitic imidazolate framework based on a Co node and 2-methyl imidazolate ligand (ZIF-67). ${ }^{9}$ While these fundamental studies demonstrate the great potential of MOFs as light harvesting and CS materials, these systems largely rely on a ligand that has limited absorption in the visible region ${ }^{7 a, 8 a, e}$ or a transition metal d-d transition with a low extinction coefficient ${ }^{9}$ as the PS, preventing their further applications in solar energy conversion. In response to these challenges, in this work, we report the excited state and CS dynamics of a porphyrin-based Ce-TCPP MOF by using optical transient absorption (OTA) and X-ray transient absorption (XTA) spectroscopy, where the TCPP ligand (tetrakis(4-carboxyphenyl)porphyrin) has broad absorption and a relatively high extinction coefficient in the UV-visible spectrum. We show that the excitation of Ce-TCPP MOFs leads to the formation of a long-lived CS state with ligand-to-metal cluster charge transfer (LCCT) character, where the mixed-phase structure in Ce-TCPP MOFs was found to play an important role in the formation of this LCCT state.

As shown in Fig. 1a, the 3D Ce-TCPP MOF was synthesized by a solvothermal reaction. The obtained product was characterized by powder XRD (Fig. 1b) and SEM (inset of Fig. 1b) and shows a needle-like shape. After comparing the XRD patterns of the Ce-TCPP MOF with the patterns simulated from the single crystal structure of many TCPP MOFs, it seems that the structure of the Ce-TCPP MOF did not agree with any of these single-phase MOFs. Instead, we found that the XRD patterns agree well with a combination of the XRD patterns of the crystal structures of VETTUK ${ }^{10}$ and CAU-19 (Fig. 1b), ${ }^{11}$ where the patterns of the former are more prominent. These results 

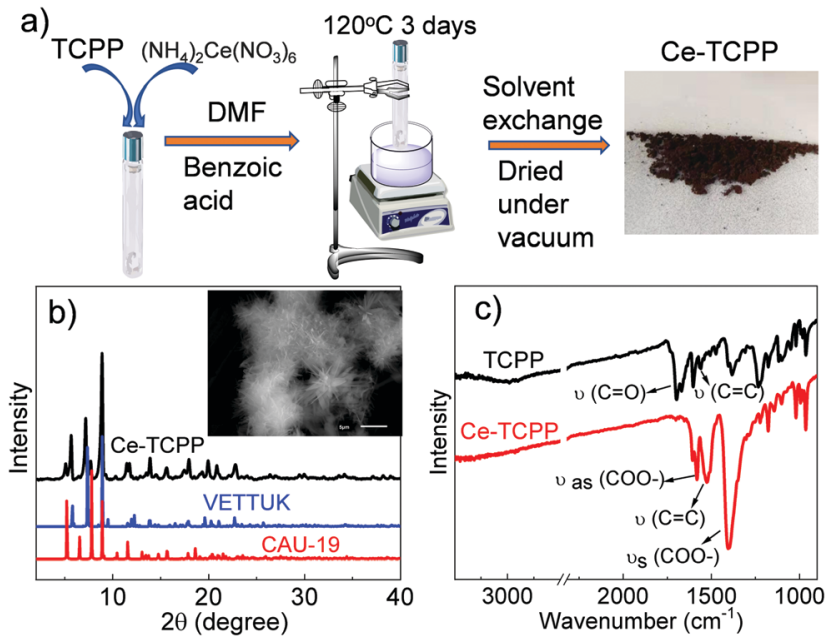

Fig. 1 (a) Synthetic scheme of Ce-TCPP; (b) XRD patterns of Ce-TCPP and patterns simulated from the single crystal structure of similar TCPP MOFs; (c) FTIR spectra of TCPP and Ce-TCPP. Inset of (b) is the SEM image of $\mathrm{Ce}-\mathrm{TCPP}$ MOFs.

suggest that the crystalline structure of Ce-TCPP MOFs is dominated by a structure similar to VETTUK with a slight contribution from CAU 19. The porosity of Ce-TCPP MOFs was confirmed by $\mathrm{N}_{2}$ sorption measurement (Fig. S1, ESI $\dagger$ ) and thermogravimetric analysis (TGA) (Fig. S2, ESI $\dagger$ ). The average BET surface area is $342.67 \mathrm{~m}^{2} \mathrm{~g}^{-1}$ based on two measurements. This value is relatively small but within the range of literature results of similar TCPP-based MOFs (330-600 $\left.\mathrm{m}^{2} \mathrm{~g}^{-1}\right){ }^{11}$ The TGA results show two characteristic steps, including a weight loss below $100{ }^{\circ} \mathrm{C}$ and one at $360-500{ }^{\circ} \mathrm{C}$, which can be attributed to the evaporation of water molecules and the decomposition of the MOF with the dissociation of the $\mathrm{Ce}-\mathrm{O}$ bond, respectively. ${ }^{11}$ The formation of Ce-TCPP MOFs was further supported by the Fourier-transform infrared (FT-IR) spectra (Fig. 1c), where we observed the disappearance of $\mathrm{C}=\mathrm{O}$ stretching at $1700 \mathrm{~cm}^{-1}$, which is present in TCPP resulting from free $-\mathrm{COOH}$, the shift of $\mathrm{C}=\mathrm{C}$ valence vibration of phenyl rings from $1559 \mathrm{~cm}^{-1}$ to $1526 \mathrm{~cm}^{-1}$, and two new peaks at $1587 \mathrm{~cm}^{-1}$ and $1400 \mathrm{~cm}^{-1}$, which can be assigned to asymmetric and symmetric vibrational stretching of $\mathrm{COO}^{-}$, respectively. ${ }^{12}$ These results together suggest the successful coordination of the carboxyl group in TCPP with the Ce metal ion in Ce-TCPP MOFs.

In addition to the bulk structure, the local coordination environment at the Ce center in Ce-TCPP MOFs was confirmed by X-ray absorption spectroscopy (XAS). The X-ray absorption near edge structure (XANES) spectra of Ce-TCPP MOFs and two reference samples, i.e., hexagonal $\mathrm{CeCl}_{3} \cdot 7 \mathrm{H}_{2} \mathrm{O}\left(\mathrm{Ce}^{3+}\right)$ and cubic $\mathrm{CeO}_{2}\left(\mathrm{Ce}^{4+}\right)$, as well as the starting material $\left(\mathrm{NH}_{4}\right)_{2} \mathrm{Ce}\left(\mathrm{NO}_{3}\right)_{6}$, are shown in Fig. 2a. The main feature at $\sim 5725.5 \mathrm{eV}$ in the spectrum of $\mathrm{CeCl}_{3}$ is the absorption white line corresponding to the dipole allowed transition from Ce $2 \mathrm{p}$ to $5 \mathrm{~d}$ mixed with the $4 \mathrm{f}^{1}$ final state, ${ }^{13}$ suggesting that $\mathrm{Ce}$ in $\mathrm{CeCl}_{3}$ is in a trivalent state $\left(\mathrm{Ce}^{3+}\right)$. In contrast, the XANES spectrum of $\mathrm{CeO}_{2}$ and $\left(\mathrm{NH}_{4}\right)_{2} \mathrm{Ce}\left(\mathrm{NO}_{3}\right)_{6}$ exhibits two distinct features, where the
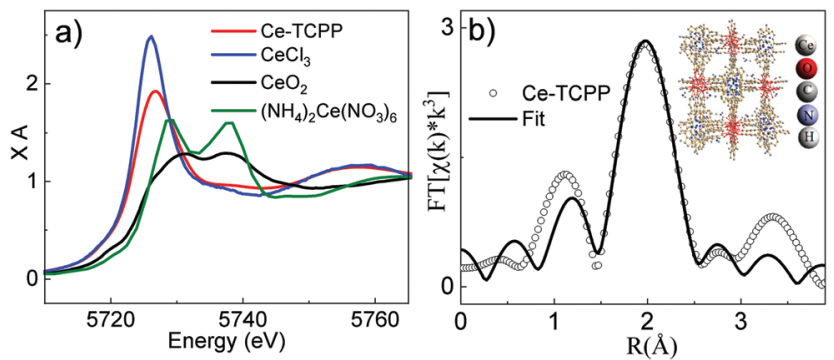

Fig. 2 (a) XANES spectra of Ce-TCPP MOFs (red), $\mathrm{CeCl}_{3}$ (blue), $\mathrm{CeO}_{2}$ (black) and $\left(\mathrm{NH}_{4}\right)_{2} \mathrm{Ce}\left(\mathrm{NO}_{3}\right)_{6}$ (olive). (b) The Fourier-transformed XAS spectrum of Ce-TCPP in $R$-space. The inset shows the fitting model. The data are shown as open dots and FEFF fits are shown as solid lines.

features for $\mathrm{CeO}_{2}$ occur at $\sim 5731 \mathrm{eV}$ and $5737.5 \mathrm{eV}$ and those for $\left(\mathrm{NH}_{4}\right)_{2} \mathrm{Ce}\left(\mathrm{NO}_{3}\right)_{6}$ occur at $5729 \mathrm{eV}$ and $5738 \mathrm{eV}$, which can be attributed to the mixed-valence behavior of tetravalent Cerium $\left(\mathrm{Ce}^{4+}\right)$ in its final state $\left(4 \mathrm{f}^{1} 5 \mathrm{dt}_{2 \mathrm{~g}} \mathrm{~L}\right.$ and $4 \mathrm{f}^{0} 5 \mathrm{~d}$; L denotes the oxygen $2 \mathrm{p}$ hole). ${ }^{14}$ Unlike the reference samples, the XANES spectrum of Ce-TCPP MOF shows the main absorption edge at $5725.5 \mathrm{eV}$ and a weak feature at $5737.5 \mathrm{eV}$, which can be assigned to the $4 \mathrm{f}^{1}$ and $4 \mathrm{f}^{0}$ absorption peaks, respectively, suggesting the co-existence of a $\mathrm{Ce}^{3+} / \mathrm{Ce}^{4+}$ valence state in the Ce-TCPP MOF. This is further supported by the energy difference $(\sim 12 \mathrm{eV})$ between these two features, which agrees well with the Coulomb interaction of Ce $2 \mathrm{p}$ and Ce $4 \mathrm{f}$ orbitals. ${ }^{15}$ As suggested by the previous literature report on similar Ce-MOFs, ${ }^{11}$ the Ce center in the Ce-TCPP MOF is dominated by $\mathrm{Ce}^{3+}$ that can be attributed to the reduction of $\mathrm{Ce}^{4+}$ by the redox-active linkers (i.e., TCPP).

To gain a deeper insight of the local coordination environment, we quantitatively fitted the extended X-ray absorption fine structure (EXAFS) spectrum of Ce-TCPP (Fig. S3a, ESI $\dagger$ ) using the Demeter XAS analysis package. As the XRD results suggest that the structure of Ce-TCPP MOF is featured by a combination of VETTUK and CAU-19, the fitting models based on the crystal structure of both VETTUK ${ }^{10}$ and CAU- $19^{11}$ were used to fit the data. It is interesting to note that the EXAFS spectrum can be adequately fit based on the crystal structure of VETTUK (fitting parameters listed in Table S1, ESI $\dagger$ ) but significant deviation was observed based on CAU-19. This can be explained by the fact that the crystalline structure of the Ce-TCPP MOF is dominated by a structure similar to VETTUK, which is consistent with the XRD results. The EXAFS data and the resulting best fit in $R$-space and $k$-space are presented in Fig. $2 \mathrm{~b}$ and Fig. $\mathrm{S} 3 \mathrm{~b}(\mathrm{ESI} \dagger)$, respectively. From the best fitting, the bond distance of Ce to $\mathrm{O}$ in Ce-TCPP was found to be between $2.22 \AA$ and $2.61 \AA$. The $\mathrm{Ce}-\mathrm{O}$ distances are within the range of distances reported in the literature for $\mathrm{Ce}(\mathrm{Iv})$ and $\mathrm{Ce}(\mathrm{III})$ clusters (2.206-2.234 $\AA$ for core Ce-O, $2.429 \AA$ to $2.563 \AA$ and 2.364 to $2.765 \AA$ for the remaining $\mathrm{Ce}-\mathrm{O}),{ }^{16}$ suggesting the validity of our fitting model.

Fig. 3a shows the UV-visible absorption spectrum of TCPP in methanol (black plot), and the diffuse reflectance (DR) spectra of TCPP in the solid state (blue plot) and Ce-TCPP MOFs (red 

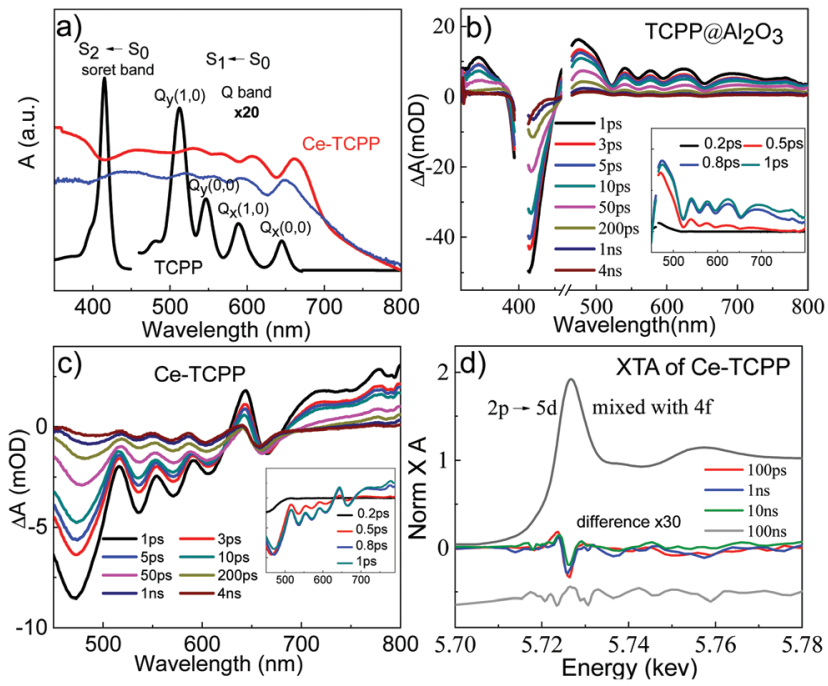

Fig. 3 (a) UV-visible absorption spectrum of TCPP in methanol and the diffuse reflectance spectrum of TCPP in the solid state (blue) and $\mathrm{Ce}-\mathrm{TCPP}$ (red). OTA spectra of TCPP/Al $\mathrm{O}_{3}$ (b) and Ce-TCPP (c) following $400 \mathrm{~nm}$ excitation. The insets show the early time OTA spectra. (d) XANES

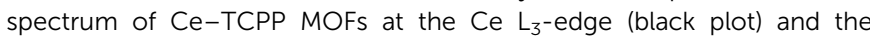
difference XANES spectra at 100 ps (red), 1 ns (blue), 10 ns (green), and 100 ns (gray) obtained by subtracting the laser-off spectrum from the laser-on spectrum. The difference spectrum at 100 ns delay is shifted manually to provide an offset for a clearer view.

plot). It was found that TCPP in methanol solution exhibits an intense Soret band centered at $415 \mathrm{~nm}\left(\mathrm{~S}_{0}-\mathrm{S}_{2}\right.$ transition) and relatively weaker $\mathrm{Q}$ bands $\left(\mathrm{S}_{0}-\mathrm{S}_{1}\right.$ transition) that spread over a wide range in the visible region with four distinct peaks at $512 \mathrm{~nm}\left(Q_{y}\right), 547 \mathrm{~nm}\left(Q_{y}\right), 588 \mathrm{~nm}\left(Q_{x}\right)$ and $645 \mathrm{~nm}\left(Q_{x}\right) \cdot{ }^{17}$ In contrast, Ce-TCPP MOFs show broad absorption extended to $\sim 700 \mathrm{~nm}$. This feature is likely due to scattering because it was also observed in the DR spectrum of TCPP in the solid state. The Soret and Q bands of Ce-TCPP MOFs show a prominent red-shift compared to TCPP, which can be attributed to the planarity change caused by the deformation of TCPP during incorporation: the confirmed non-planarity of TCPP in the Ce-TCPP MOF destabilizes the porphyrin HOMOs while the LUMOs were not significantly affected. ${ }^{18}$ Moreover, the strong coupling of the well-arranged TCPP monomers (J-aggregation $)^{17 b}$ may also contribute to the red-shift, which has been observed in other porphyrin-based MOFs reported previously. ${ }^{19}$

Femtosecond OTA spectroscopy was performed with a selective excitation of the TCPP Soret band to examine the excited state (ES) dynamics of the Ce-TCPP MOFs. To better understand the effect of the porous nature on the ES dynamics in the MOFs, we first measured the OTA spectra of TCPP on an $\mathrm{Al}_{2} \mathrm{O}_{3}$ thin film, which represents a control sample for intrinsic ES dynamics of TCPP in a heterogeneous environment. As shown in Fig. $3 \mathrm{~b}$ and Fig. S4a (ESI $\dagger$ ), the OTA spectra of TCPP/ $/ \mathrm{Al}_{2} \mathrm{O}_{3}$ thin film consist of a negative band centered at $415 \mathrm{~nm}$ and a broad positive absorption feature from $450 \mathrm{~nm}$ overlapping with several distinct bleach signals at $520 \mathrm{~nm}, 563 \mathrm{~nm}, 596 \mathrm{~nm}$ and $653 \mathrm{~nm}$. These features have been well studied and can be attributed to the TCPP Soret band ground state bleach (GSB), ES absorption (ESA), and Q band GSB, respectively. The recovery of the Soret GSB and the decay of ES follow the same kinetics (Fig. S4b, ESI $\dagger$ ), together with the presence of the isosbestic point at $451 \mathrm{~nm}$, suggesting that the decay of ES molecules to their GS is the only relaxation process in $\mathrm{TCPP} / \mathrm{Al}_{2} \mathrm{O}_{3}$ after excitation.

Compared to TCPP, the OTA spectra of Ce-TCPP MOFs are dramatically different. As shown in Fig. 3c, the OTA spectra of Ce-TCPP exhibit the Soret and Q band GSB with a red-shift in the range of $450 \mathrm{~nm}$ to $670 \mathrm{~nm}$, which is consistent with its UV-visible ground state DR reflectance spectrum. However, the positive features pertaining to ESA are missing in the region $<600 \mathrm{~nm}$ even at early times ( $<1 \mathrm{ps}$ ) (inset of Fig. 3c). We attribute this difference to the ultrafast $(<200 \mathrm{fs})$ formation of a new CS state evolved from singlet ES (i.e., $\mathrm{S}_{2}$ and $\mathrm{S}_{1}$ ) (Fig. S4c, ESI $\dagger$ ), as superfast CS was also observed upon $Q$ band excitation (Fig. S4d, ESI $\dagger$ ). The CS was further confirmed by observation of a transient feature in the region $>700 \mathrm{~nm}$ as the fingerprint absorption of one electron oxidized $\mathrm{TCPP}^{*+} .{ }^{20}$ These results, similar to previous reports accounting for electron transfer (ET) processes in MOFs, ${ }^{19,21}$ imply that fast ET occurs from TCPP ligand to Ce metal cluster.

To gain more insight on the nature of the CS state, we measured the OTA spectra of three reference samples including CAU-19, a Ce-based MOF that has the same crystal structure as VETTUK (denoted as Ce-VETTUK), and a physical mixture of these two MOFs. The details of the synthetic procedure and structural characterization (XRD, FTIR, DR spectrum, and XAS) of these two MOFs are presented in the ESI and Fig. S5-S8 (ESI $\dagger$ ). The OTA spectra of CAU-19 (Fig. S9a, ESI $\dagger$ ), Ce-VETTUK (Fig. S9b, ESI $\dagger$ ), and the physical mixture of both MOFs (Fig. S9c, ESI $\dagger$ ) largely look similar to that of $\mathrm{TCPP} / \mathrm{Al}_{2} \mathrm{O}_{3}$, consisting of a Soret GSB at $<480 \mathrm{~nm}$, broad ESA, and multiple Q band GSB signals. However, it is notable that the Q band GSB bands, which remain positive in the spectra of TCPP $/ \mathrm{Al}_{2} \mathrm{O}_{3}$ at all time delays, become negative in the spectra of CAU-19 at $\sim 100 \mathrm{ps,}$ Ce-VETTUK at $\sim 50 \mathrm{ps}$, and the physical mixture of CAU-19 and Ce-VETTUK as early as 1 ps (Fig. S9 and S10, ESI $\dagger$ ). These results together suggest that the CS state observed in the mixed phase Ce-TCPP MOF also occurs in these reference samples, where the formation of CS is fastest in the mixed-phase Ce-TCPP, which then decreases following the order of the physical mixture of CAU-19 and Ce-VETTUK > Ce-VETTUK > CAU-19 (Fig. S11, ESI $\dagger$ ). These results suggest that the mixedphase crystal structure in Ce-TCPP plays an important role in facilitating CS. While the specific roles of each phase remain unclear, similar phase facilitated CS has been observed in other mixed-phase materials. ${ }^{22}$ Nevertheless, we can conclude that the presence of a long-lived CS state after ultrafast ET in the mixed-phase Ce-TCPP is expected to be beneficial for applications in photocatalysis.

To gain further insight on the nature of the long-lived CS in the mixed-phase Ce-TCPP, we directly probed the electron density changes at the Ce center following selective excitation of the TCPP ligand using XTA spectroscopy. Fig. 3d shows the 
XANES spectrum of Ce-TCPP at the Ce L3 edge and the difference spectra obtained by subtracting the GS (laser-off) spectrum from the spectrum collected at different delay times (100 ps, $1 \mathrm{~ns}, 10 \mathrm{~ns}$ and $100 \mathrm{~ns}$ ) following $400 \mathrm{~nm}$ laser excitation. The positive feature observed at $5723.7 \mathrm{eV}$ indicates that the edge energy of Ce shifts to a lower energy, suggesting the formation of a reduced Ce center in Ce-TCPP. This is further supported by the negative feature observed at $5725.5 \mathrm{eV}$ : the decreased number of empty $4 \mathrm{f}$ orbitals prohibits the excitation of $2 \mathrm{p}$ core electrons, resulting in the decreased absorption intensity. The intensity of this negative feature decreases gradually from 100 ps to $10 \mathrm{~ns}$ until 100 ns where no transient signal was observed, suggesting that this is a long-lived transient species ( $>10 \mathrm{~ns}$ ). These results together confirmed the formation of a long-lived CS state due to ligand-to-metal cluster charge transfer (LCCT) after photoexcitation of Ce-TCPP, consistent with the OTA results above. As the Ce centers in Ce-TCPP are dominated by $\mathrm{Ce}^{3+}$, which is a stable oxidation state, the nature of the long-lived CS state is likely the reduction of a small fraction of $\mathrm{Ce}^{4+}$ by the TCPP ligands, which is similar to the mechanism of forming $\mathrm{Ce}^{3+}$ centers in Ce-TCPP MOF from $\mathrm{Ce}^{4+}$ in the starting material, $\left(\mathrm{NH}_{4}\right)_{2} \mathrm{Ce}\left(\mathrm{NO}_{3}\right)_{6}{ }^{23}$

In summary, we have synthesized mixed-phase Ce-TCPP constructed from the free-base TCPP ligand and Cerium ammonium nitrate. Using OTA spectroscopy, we show that ultrafast ET occurs from the TCPP ligand to Ce center in Ce-TCPP MOFs following excitation of the TCPP ligand, forming a long-lived CS state. Systematic OTA studies on three control samples revealed that the presence of a mixed-phase structure in the Ce-TCPP MOF is responsible for the ultrafast formation of the $\mathrm{CS}$ state. The nature of this CS state is LCCT, as confirmed by XTA, where the reduction of Ce centers was observed due to excitation of the TCPP ligand. The observed ultrafast charge transfer process that results in the formation of a long-lived CS state ( $>10 \mathrm{~ns}$ ) is expected to be beneficial for photocatalysis and thus implies the potential application of Ce-TCPP MOFs in solar energy conversion.

This work was supported by the National Science Foundation (CBET-1706971). Use of the Advanced Photon Source at Argonne National Laboratory was supported by the U.S. Department of Energy, Office of Science, Office of Basic Energy Sciences, under Award No. DE-AC02-06CH11357. Sizhuo Yang acknowledges the John J. Eisch fellowship during the 2018-2019 academic year.

\section{Conflicts of interest}

There are no conflicts to declare.

\section{Notes and references}

1 (a) M. Aresta, A. Dibenedetto and A. Angelini, Chem. Rev., 2014, 114, 1709-1742; (b) T. Sakakura, J.-C. Choi and H. Yasuda, Chem. Rev., 2007, 107, 2365-2387.

2 (a) P. Moroz, L. Royo Romero and M. Zamkov, Chem. Commun., 2019, 55, 3033-3048; (b) S. Ye, C. Ding, M. Liu, A. Wang, Q. Huang and C. Li, Adv. Mater., 2019, 31, 1902069; (c) S. Ye, C. Ding, R. Chen, F. Fan, P. Fu, H. Yin, X. Wang, Z. Wang, P. Du and C. Li, J. Am. Chem. Soc., 2018, 140, 3250-3256; (d) P. Kang, C. Cheng, Z. Chen, C. K. Schauer, T. J. Meyer and M. Brookhart, J. Am. Chem. Soc., 2012, 134, 5500-5503; (e) P. Hu, Y. Diskin-Posner, Y. Ben-David and D. Milstein, ACS Catal., 2014, 4, 2649-2652.
3 H. Furukawa, K. E. Cordova, M. O'Keeffe and O. M. Yaghi, Science, 2013, 341, 1230444.

4 (a) S. Yang, B. Pattengale, S. Lee and J. Huang, ACS Energy Lett., 2018, 3, 532-539; (b) C. Wang, Z. Xie, K. E. deKrafft and W. Lin, J. Am. Chem. Soc., 2011, 133, 13445-13454; (c) M. Lammert, M. T. Wharmby, S. Smolders, B. Bueken, A. Lieb, K. A. Lomachenko, D. D. Vos and N. Stock, Chem. Commun., 2015, 51, 12578-12581.

5 (a) A. Dhakshinamoorthy, Z. H. Li and H. Garcia, Chem. Soc. Rev., 2018, 47, 8134-8172; (b) J.-D. Xiao and H.-L. Jiang, Acc. Chem. Res., 2019, 52, 356-366.

6 (a) N. Kornienko, Y. Zhao, C. S. Kley, C. Zhu, D. Kim, S. Lin, C. J. Chang, O. M. Yaghi and P. Yang, J. Am. Chem. Soc., 2015, 137, 14129-14135; (b) R. Li, W. Zhang and K. Zhou, Adv. Mater., 2018, 30, 31; (c) A. M. Rice, G. A. Leith, O. A. Ejegbavwo, E. A. Dolgopolova and N. B. Shustova, ACS Energy Lett., 2019, 4, 1938-1946.

7 (a) L. Hanna, P. Kucheryavy, C. Liu, X. Zhang and J. V. Lockard, J. Phys. Chem. C, 2017, 121, 13570-13576; (b) S. M. Shaikh, A. Chakraborty, J. Alatis, M. Cai, E. Danilov and A. J. Morris, Faraday Discuss., 2019, 216, 174-190.

8 (a) L. Hanna, C. L. Long, X. Zhang and J. V. Lockard, Chem. Commun., 2020, 56, 11597-11600; (b) E. Caballero-Mancebo, B. Cohen, S. Smolders, D. E. De Vos and A. Douhal, Adv. Sci., 2019, 6, 1901020; (c) J. Yu, J. Park, A. Van Wyk, G. Rumbles and P. Deria, J. Am. Chem. Soc., 2018, 140, 10488-10496; (d) M. Alvaro, E. Carbonell, B. Ferrer, F. X. LlabrésiXamena and H. Garcia, Eur. J. Chem., 2007, 13, 5106-5112; (e) M. de Miguel, F. Ragon, T. Devic, C. Serre, P. Horcajada and H. García, ChemPhysChem, 2012, 13, 3651-3654.

9 B. Pattengale, S. Yang, J. Ludwig, Z. Huang, X. Zhang and J. Huang, J. Am. Chem. Soc., 2016, 138, 8072-8075.

10 S. George, S. Lipstman and I. Goldberg, Cryst. Growth Des., 2006, 6, 2651-2654.

11 T. Rhauderwiek, N. Heidenreich, H. Reinsch, S. Oien-Odegaard, K. A. Lomachenko, U. Rutt, A. V. Soldatov, K. P. Lillerud and N. Stock, Cryst. Growth Des., 2017, 17, 3462-3474.

12 A. Lin, A. A. Ibrahim, P. Arab, H. M. El-Kaderi and M. S. El-Shall, ACS Appl. Mater. Interfaces, 2017, 9, 17961-17968.

13 T. Sugimoto, B. Joseph, E. Paris, A. Iadecola, T. Mizokawa, S. Demura, Y. Mizuguchi, Y. Takano and N. L. Saini, Phys. Rev. B: Condens. Matter Mater. Phys., 2014, 89.

14 G. Jacobs, P. M. Patterson, L. Williams, E. Chenu, D. Sparks, G. Thomas and B. H. Davis, Appl. Catal., A, 2004, 262, 177-187.

15 (a) B. T. Thole, G. Vanderlaan, J. C. Fuggle, G. A. Sawatzky, R. C. Karnatak and J. M. Esteva, Phys. Rev. B: Condens. Matter Mater. Phys., 1985, 32, 5107-5118; (b) A. Kotani, K. O. Kvashnina, P. Glatzel, J. C. Parlebas and G. Schmerber, Phys. Rev. Lett., 2012, 108, 5.

16 (a) L. Mathey, M. Paul, C. Coperet, H. Tsurugi and K. Mashima, Chem. - Eur. J., 2015, 21, 13454-13461; (b) R. Das, R. Sarma and J. B. Baruah, Inorg. Chem. Commun., 2010, 13, 793-795.

17 (a) Y. Venkatesh, M. Venkatesan, B. Ramakrishna and P. R. Bangal, J. Phys. Chem. B, 2016, 120, 9410-9421; (b) B. D. McCarthy, E. R. Hontz, S. R. Yost, T. Van Voorhis and M. Dincă, J. Phys. Chem. Lett., 2013, 4, 453-458.

18 (a) A. B. J. Parusel, T. Wondimagegn and A. Ghosh, J. Am. Chem. Soc., 2000, 122, 6371-6374; (b) R. E. Haddad, S. Gazeau, J. Pecaut, J. C. Marchon, C. J. Medforth and J. A. Shelnutt, J. Am. Chem. Soc., 2003, 125, 1253-1268.

19 X. X. Li, C. H. Gong, G. G. Gurzadyan, M. F. Gelin, J. X. Liu and L. C. Sun, J. Phys. Chem. C, 2018, 122, 50-61.

20 (a) A. Harriman, J. Chem. Soc., Faraday Trans., 1981, 77, 1281-1291; (b) P. M. Keane and J. M. Kelly, Photochem. Photobiol. Sci., 2011, 10, 1578-1586.

21 M. Gutierrez, B. Cohen, F. Sanchez and A. Douhal, Phys. Chem. Chem. Phys., 2016, 18, 27761-27774.

22 (a) A. Li, Z. Wang, H. Yin, S. Wang, P. Yan, B. Huang, X. Wang, R. Li, X. Zong, H. Han and C. Li, Chem. Sci., 2016, 7, 6076-6082; (b) N. A. Rodríguez, R. Parra and M. A. Grela, RSC Adv., 2015, 5, 73112-73118; (c) A. Henning, V. K. Sangwan, H. Bergeron, I. Balla, Z. Sun, M. C. Hersam and L. J. Lauhon, ACS Appl. Mater. Interfaces, 2018, 10, 16760-16767; (d) T. Ferron, M. Waldrip, M. Pope and B. A. Collins, J. Mater. Chem. A, 2019, 7, 4536-4548.

23 (a) Cerium and Cerium Compounds. In Kirk-Othmer Encyclopedia of Chemical Technology, pp. 1-23; (b) E. J. Schelter, Nat. Chem., $2013,5,348$. 